\title{
Morfologia e biometria do timo em araras dos gêneros Ara e Anodorhynchus
}

\author{
[Morphology and biometry of the thymus gland in macaw of Ara and Anodorhynchus genera] \\ R.Z. Lima ${ }^{1}$, E.S. Gonçalves ${ }^{1}$, M.I. Santana ${ }^{2 *}$, E.M.M. Lima ${ }^{2}$, F.O.C. Silva ${ }^{3}$, \\ R.S. Severino ${ }^{3}$, S.S. Drummond ${ }^{3}$, A.P.R.N. Armando ${ }^{4}$ \\ ${ }^{1}$ PUC Minas - Poços de Caldas, MG \\ ${ }^{2}$ Universidade de Brasília - Brasília, DF \\ ${ }^{3}$ Universidade Federal de Uberlândia - Uberlândia, MG \\ ${ }^{4}$ Criadouro Poços de Caldas - Poços de Caldas, MG
}

\begin{abstract}
RESUMO
Foram utilizados 12 exemplares de Ara ararauna - seis fêmeas e seis machos -, cinco exemplares de Ara chloropterus (uma fêmea e quatro machos) e dois exemplares de Anodorhynchus hyacinthinus - uma fêmea e um macho -, todos adultos, doados por criadouro particular, após óbito natural. Os lobos foram dissecados e medidos com paquímetro - comprimento $\mathrm{x}$ largura $\mathrm{x}$ espessura - e analisados quanto ao peso, à topografia e à morfologia individual. Independentemente do gênero, foram identificados, em 17 casos $(89,5 \%)$, lobos tímicos nos antímeros cervicais esquerdo e direito, e em oito casos $(42,1 \%)$, lobos na cavidade celomática. Os lobos apresentaram formatos alongados $-52,6 \%-$, arredondados $-21,1 \%-$ ou ovalados - 15,8\% -, posicionados preferencialmente ventromedialmente ao longo do plexo vasculoneural do pescoço, com número médio de cinco lobos por antímero, tamanho médio de $0,49 \mathrm{~cm}$ de comprimento, $0,12 \mathrm{~cm}$ de largura e $0,05 \mathrm{~cm}$ de espessura e peso médio de $0,076 \mathrm{~g}$.
\end{abstract}

Palavras-chave: araras, anatomia comparada, aves silvestres, morfologia do timo

\begin{abstract}
Twelve samples of Ara ararauna - six females and six males -, five samples of Ara chloropterus (one female and four males) and two samples of Anodorhynchus hyacinthinus (one female and one male), all adults from a Breeding Park, were used after natural death. The lobes were dissected and measured with electronic calliper (length $x$ width $x$ thickness) and analyzed taking their weight, topography and individual morphology into account. Regardless of gender, $89.5 \%$ of the cases presented timic lobes in the left and right cervical antimere, and $42.1 \%$ of the cases presented lobes in the celomatic cavity. The lobes were shown in shapes - long (52.6\%), round (21.1\%) or oval (15.8\%), positioned mostly ventromedially, along the neurovascular plexus of the neck, with an average of five lobes per antimere, and an average size of $0.49 \mathrm{~cm}$ length, $0.12 \mathrm{~cm}$ width, and $0.05 \mathrm{~cm}$ thick and average weight of $0.076 \mathrm{~g}$.
\end{abstract}

Keywords: Macaws, compared anatomy, wild birds, thymus gland morphology

\section{INTRODUÇÃO}

O estudo morfológico do timo tem sido objeto de intenso esforço investigativo (Ercolini, 2007), a fim de relacioná-lo com diversos aspectos concernentes ao sistema imunitário, focando principalmente os diferentes mecanismos de aquisição de defesas orgânicas (Bombonato, 1997).

Recebido em 2 de setembro de 2011

Aceito em 20 de julho de 2012

* Autor para correspondência (corresponding author)

E-mail: misantana@unb.br
A função tímica é responsável por tais mecanismos, contudo ainda não é totalmente esclarecida, tampouco são conhecidas as bases morfológicas que respondem por tais funções, Além disso, seu processo de desenvolvimento e involução ainda está por ser respondido (Agreste et al., 2007).

Aspectos anatômicos do timo de aves pertencentes à avifauna brasileira foram pouco estudados; assim, perguntas básicas deixam de ser respondidas, como seus aspectos 
comparativos referentes à topografia, à biometria e ao número de lobos (Santana et al., 2007). Além do mais, dados morfológicos, como tamanho e peso dos órgãos linfoides, podem ser utilizados como parâmetros para a avaliação da imunocompetência individual, sendo que o tamanho do timo é um excelente indicador do estado de saúde das aves (Marín et al., 2004).

Em decorrência disto, o objetivo deste trabalho foi estudar a anatomia macroscópica - número, topografia, morfologia e biometria dos lobos tímicos - de três espécies de araras brasileiras (Ara ararauna, Ara chloroptera $e$ Anodorhynchus hyacinthinus).

\section{MATERIAIS E MÉTODOS}

Foram utilizados 12 exemplares de ararascanindés (Ara ararauna), seis fêmeas e seis machos; cinco araras-vermelhas (Ara chloropterus), uma fêmea e quatro machos, e duas araras-azuis (Anodorhynchus hyacinthinus), uma fêmea e um macho, todas adultas e com pesos variados, oriundas do Criadouro Poços de Caldas, localizado na cidade de Poços de Caldas, MG. As aves foram doadas após óbito natural e preparadas em Laboratório Escola de Anatomia Veterinária.

Após o recebimento, as araras foram imediatamente dissecadas, inicialmente por meio de uma incisão mediana ventral na pele da região cervical, com seu rebatimento dorsal, além da retirada dos tecidos adiposo e conjuntivo que recobriam os lobos tímicos. Em seguida, procedeu-se à remoção do osso esterno juntamente com os músculos peitorais, seccionando as paredes laterais da cavidade toracoabdominal, incidindo as costelas, os ossos coracoides e a clavícula, além das porções laterocraniais dos músculos peitorais, resultando na exposição da cavidade celomática, o que facilitou a observação dos lobos situados nesta cavidade.

Para a descrição dos lobos tímicos, o órgão foi dividido em porções cervicais e celomáticas, uma direita e outra esquerda, as quais foram dissecadas com material cirúrgico convencional, valendo, quando necessário, do campo visual de uma lupa Nikon® SMZ645, modelo C-DS.

Para a realização da biometria do órgão, as mensurações dos lobos tímicos foram feitas com paquímetro Starret $^{\circledR}$. Para a padronização das medidas, optou-se pela obtenção do comprimento - eixo craniocaudal, da largura eixo dorsoventral -, e da espessura - eixo laterolateral - dos lobos tímicos. Para a contagem e o posicionamento dos lobos tímicos, sempre foi considerado o lobo mais cranial como o primeiro. A pesagem foi realizada em balança analítica $(0,0001 \mathrm{~g})$, após a retirada de toda a camada de tecido adiposo que recobria os lobos.

A nomenclatura anatômica utilizada para designação das estruturas tratadas neste trabalho está de acordo com a Nomina Anatomica Avium (Baumel, 1993).

\section{RESULTADOS}

Independentemente do gênero (Ara, Anodorhynchus), as porções cervical e celomática do timo foram identificadas em 17 casos $(89,5 \%)$ e oito casos $(42,1 \%)$, respectivamente, apresentando lobos individualizados e separados por tecido adiposo, com tamanhos diferenciados e formas irregulares. Em 10 eram alongados (52,6\%), em quatro arredondados $(21,1 \%)$ e em três ovalados $(15,8 \%)$. Não foram encontrados lobos cervicais em dois casos $(10,5 \%)$ e lobos celomáticos em $11(57,9 \%)$.

Quando presentes, os lobos cervicais puderam ser observados, no antímero esquerdo, desde o osso clavicular até o terço médio do pescoço em seis casos $(31,6 \%)$, e em um $(5,3 \%)$, no direito (Fig. 1). Também foram observados lobos cervicais apenas no terço cranial do pescoço em 11 casos $(57,9 \%)$, no antímero esquerdo, e em 16 $(84,2 \%)$, no antímero direito.

Os lobos celomáticos foram encontrados sempre caudalmente ao osso clavicular, sendo observados em três casos $(15,8 \%)$ em ambos os antímeros; quatro $(21,0 \%)$ apenas no antímero direito e um $(5,3 \%)$ apenas no antímero esquerdo.

Os lobos tímicos cervicais presentes no antímero esquerdo estiveram sempre em íntima relação com a artéria comum do nervo vago, juntamente com o nervo vago e a veia jugular, já que estavam envoltos por uma delgada membrana de tecido conjuntivo e adiposo (Fig. 2), formando, assim, um fascículo subcutâneo. 


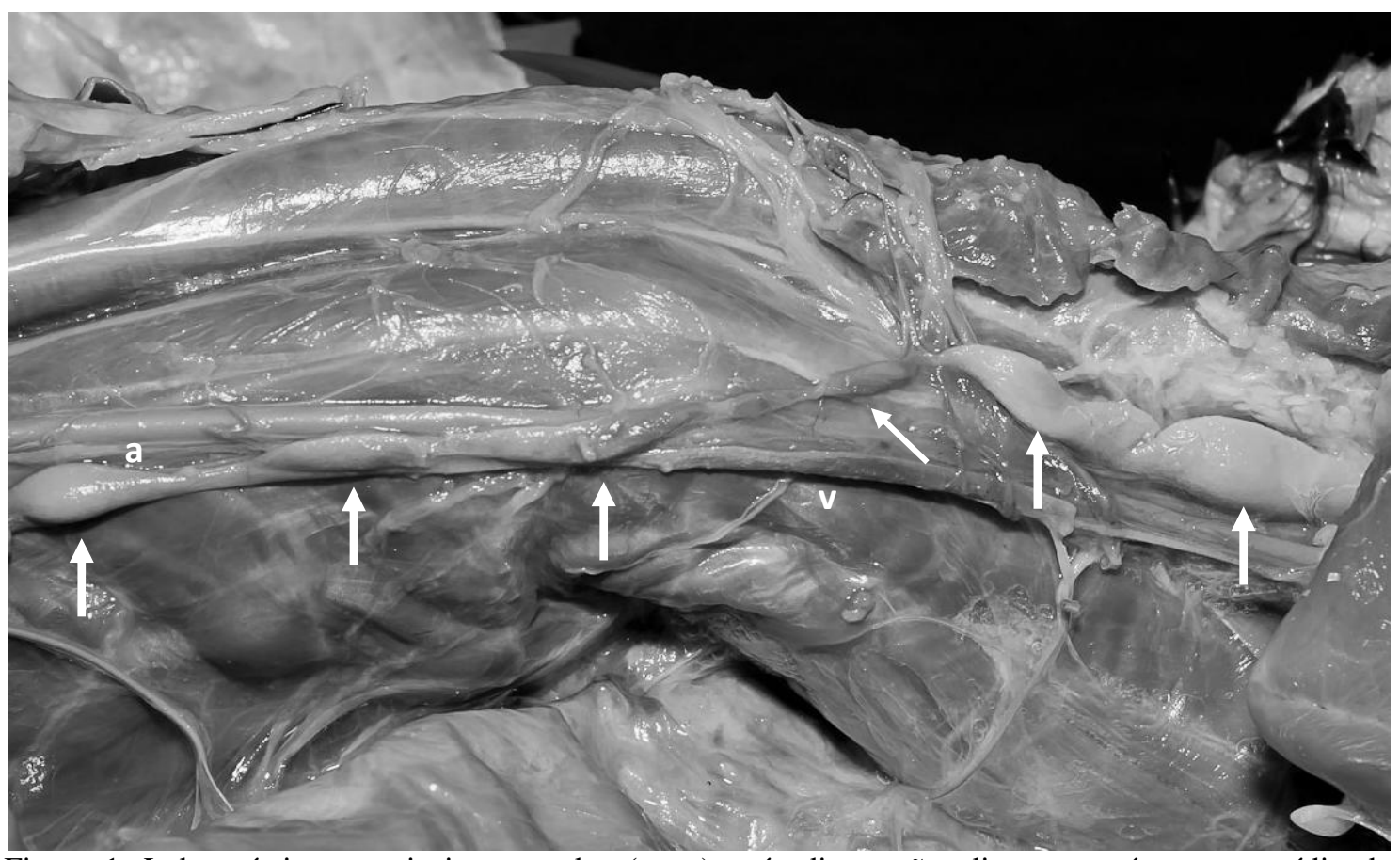

Figura 1. Lobos tímicos cervicais esquerdos (setas) após dissecação, dispostos até o terço médio do pescoço (cranial à direita), em íntima relação com a artéria comum do nervo vago (a) e da veia jugular (v).

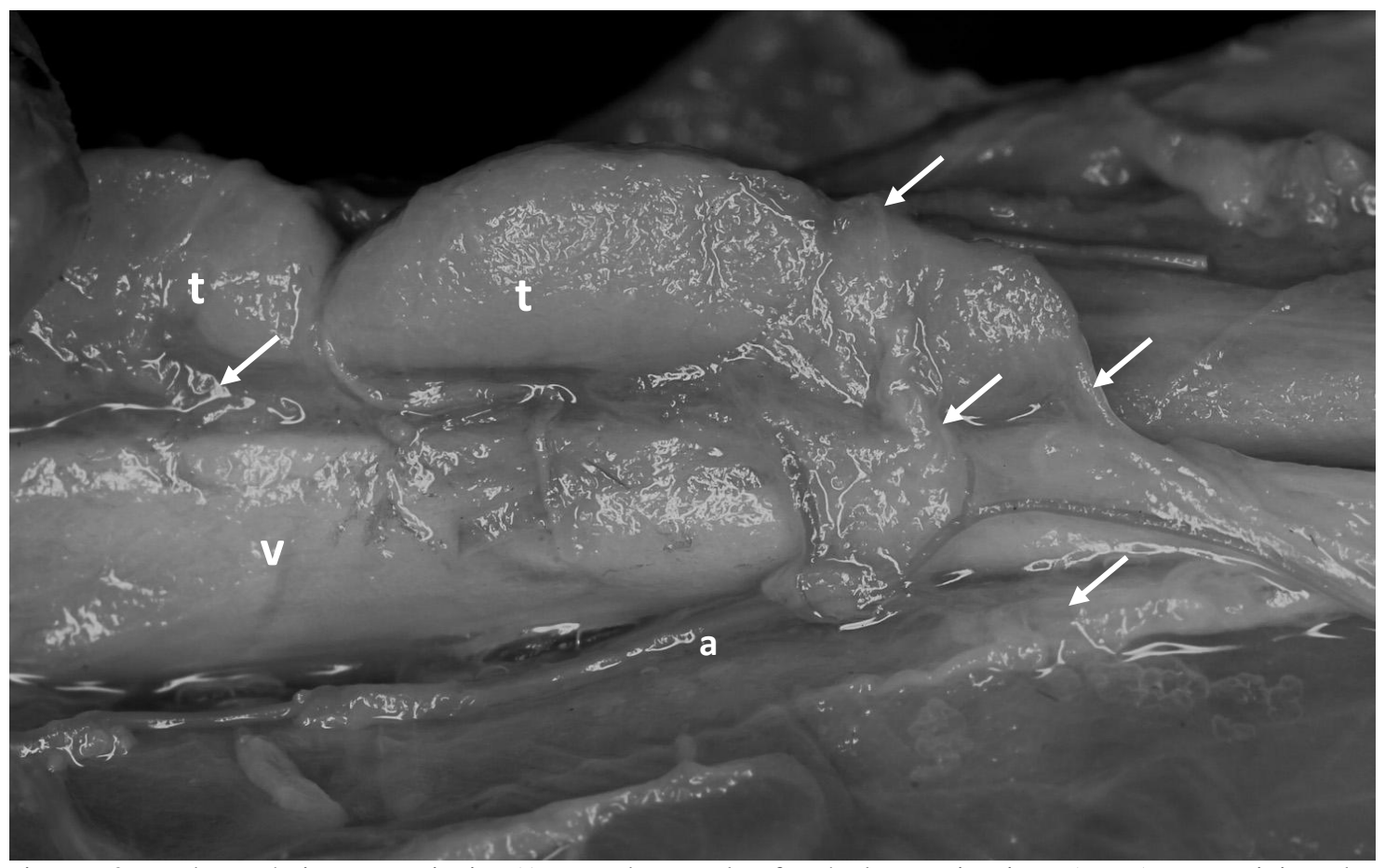

Figura 2. Lobos tímicos cervicais (t) envoltos pelo fascículo conjuntivo (setas) e posicionados ventromedialmente à veia jugular (v) e à artéria comum do nervo vago (a). 
No antímero direito, esta relação também foi observada, apesar da ausência da artéria comum do nervo vago em todas as aves estudadas, sendo a associação feita apenas com a veia jugular direita e o nervo vago. Os lobos localizados na cavidade celomática não apresentaram relação com o fascículo subcutâneo, sendo apenas recobertos dorsalmente pelo saco aéreo clavicular. Em ambos os antímeros, o fascículo subcutâneo estava localizado dorsolateralmente ao pescoço, estando relacionado com o esôfago e o inglúvio no antímero direito e medialmente com a traqueia no antímero esquerdo.

Ainda, foi notada a presença da glândula tireoide penetrando o parênquima tímico, sendo este fato observado em dois casos (10,5\%) em ararascanindés e em um caso em arara-azul.

Quanto ao número de lobos cervicais, independentemente do gênero, variou de zero a nove para o antímero direito, média de 4,6 , e de zero a 11 para o esquerdo, média de 4,7. Quando considerado o gênero, foi encontrado em ararascanindés variação de cinco a oito lobos para o antímero direito, média de 5,7 , e de cinco a 10 lobos para o esquerdo, média de 6,4. Para as araras-vermelhas, o número de lobos direitos variou de zero a seis, média de 2,6 , e de zero a cinco para os lobos esquerdos, média de 2,0, enquanto para as araras-azuis, o número de lobos direitos variou de três a quatro lobos, média de 3,5, e de zero a dois lobos esquerdos, média de 1,0 (Fig. 3).

O número de lobos celomáticos, independentemente do gênero, variou de zero a três para o antímero direito, média de 0,84 , e de zero a dois para o antímero esquerdo, média de 0,26 . Quanto ao gênero, observou-se, para as araras-canindés, o número de zero a três lobos para o antímero direito, média de 0,63 , e de zero a dois lobos para o antímero esquerdo, média de 0,26 . Nas araras-azuis, foi encontrado apenas um lobo no antímero esquerdo, enquanto nas ararasvermelhas não foram encontrados lobos cavitários (Fig. 4).

Para a posição dos lobos tímicos cervicais frente ao plexo neurovascular do pescoço, ficou estabelecido para todos os gêneros de araras estudados que as posições ventromedial (VM) e ventrolateral (VL) foram as mais frequentes, seguidas pelas dorsomedial (DM) e dorsolateral (DL), sendo o mesmo quadro observado para os lobos tímicos celomáticos (Fig. 5 e 6).

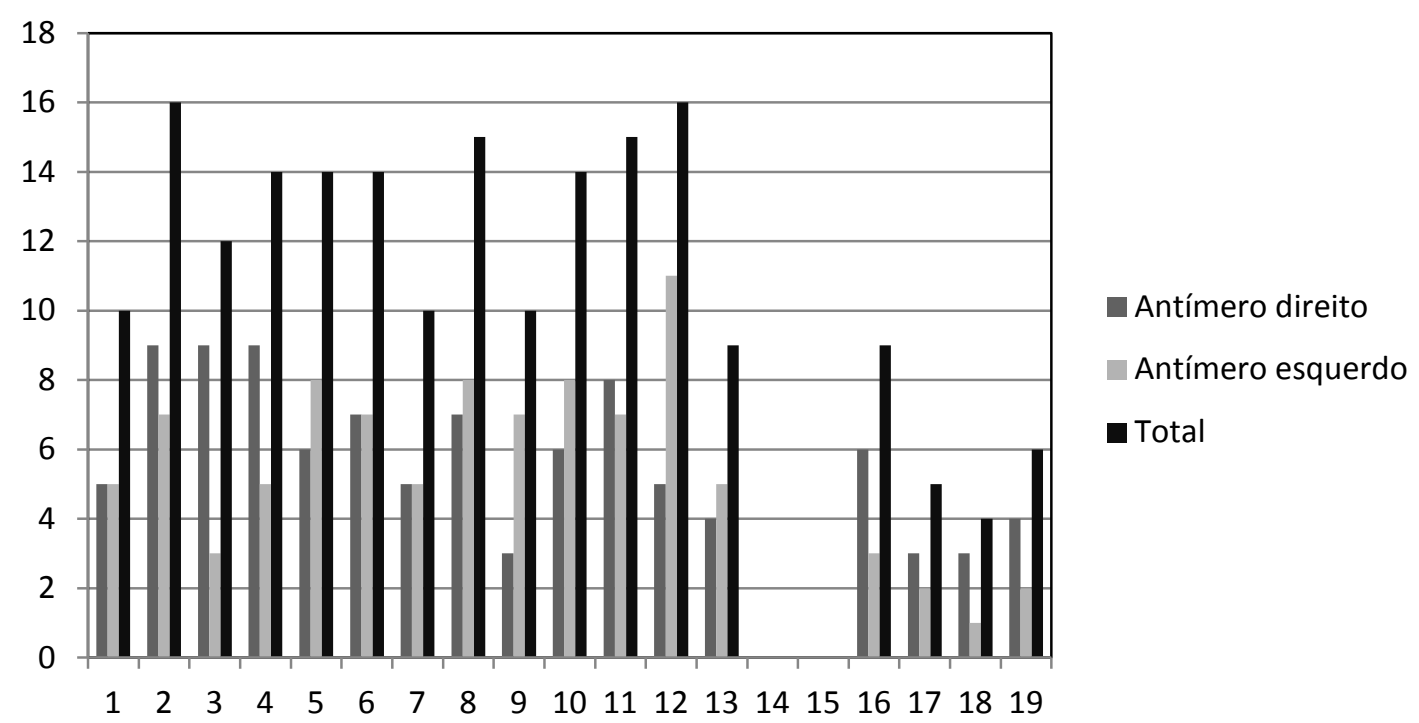

Figura 3. Número de lobos tímicos cervicais totais e por antímero. Os números de um a 12 correspondem às araras da espécie Ara arauna, de 13 a 17 às araras da espécie Ara chloropterus e de 18 a 19 às araras da espécie Anodorhynchus hyacinthinus. 


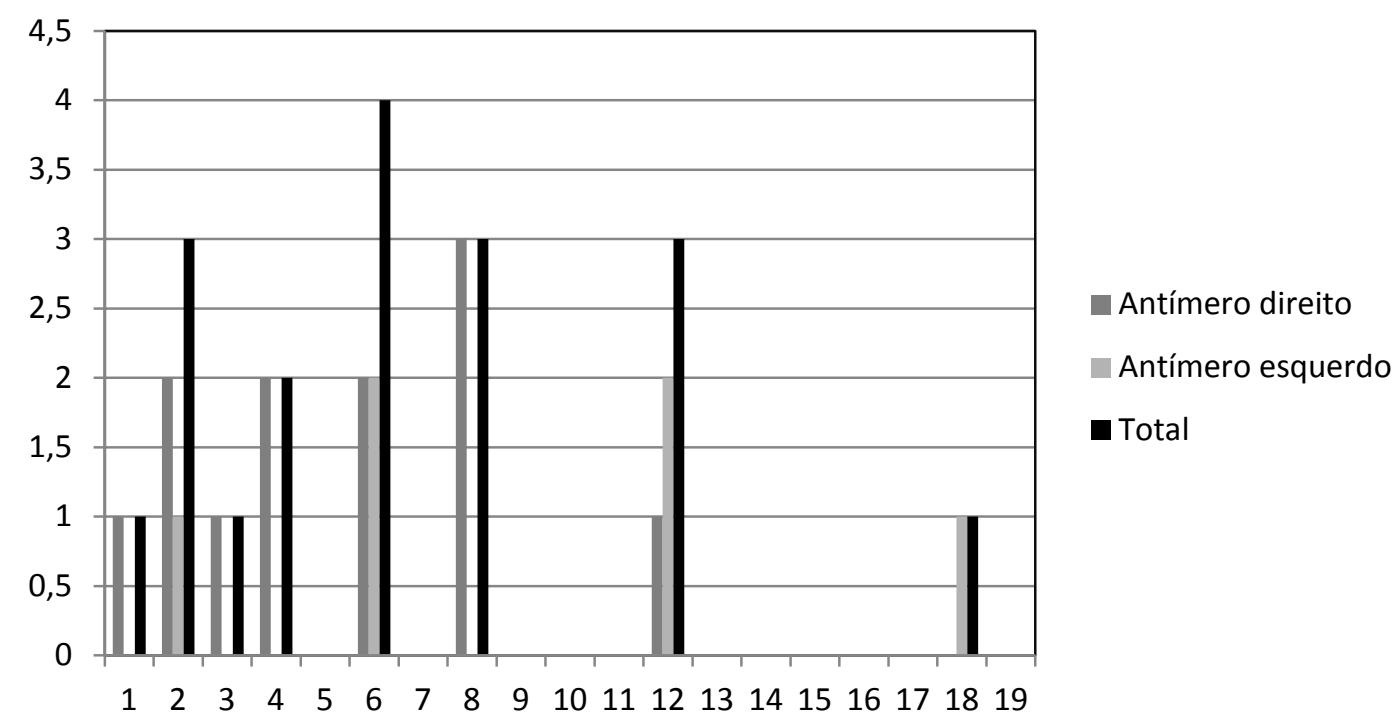

Figura 4. Número de lobos tímicos celomáticos totais e por antímero. Os números de um a 12 correspondem às araras da espécie Ara arauna, de 13 a 17 às araras da espécie Ara chloropterus e de 18 a 19 às araras da espécie Anodorhynchus hyacinthinus.

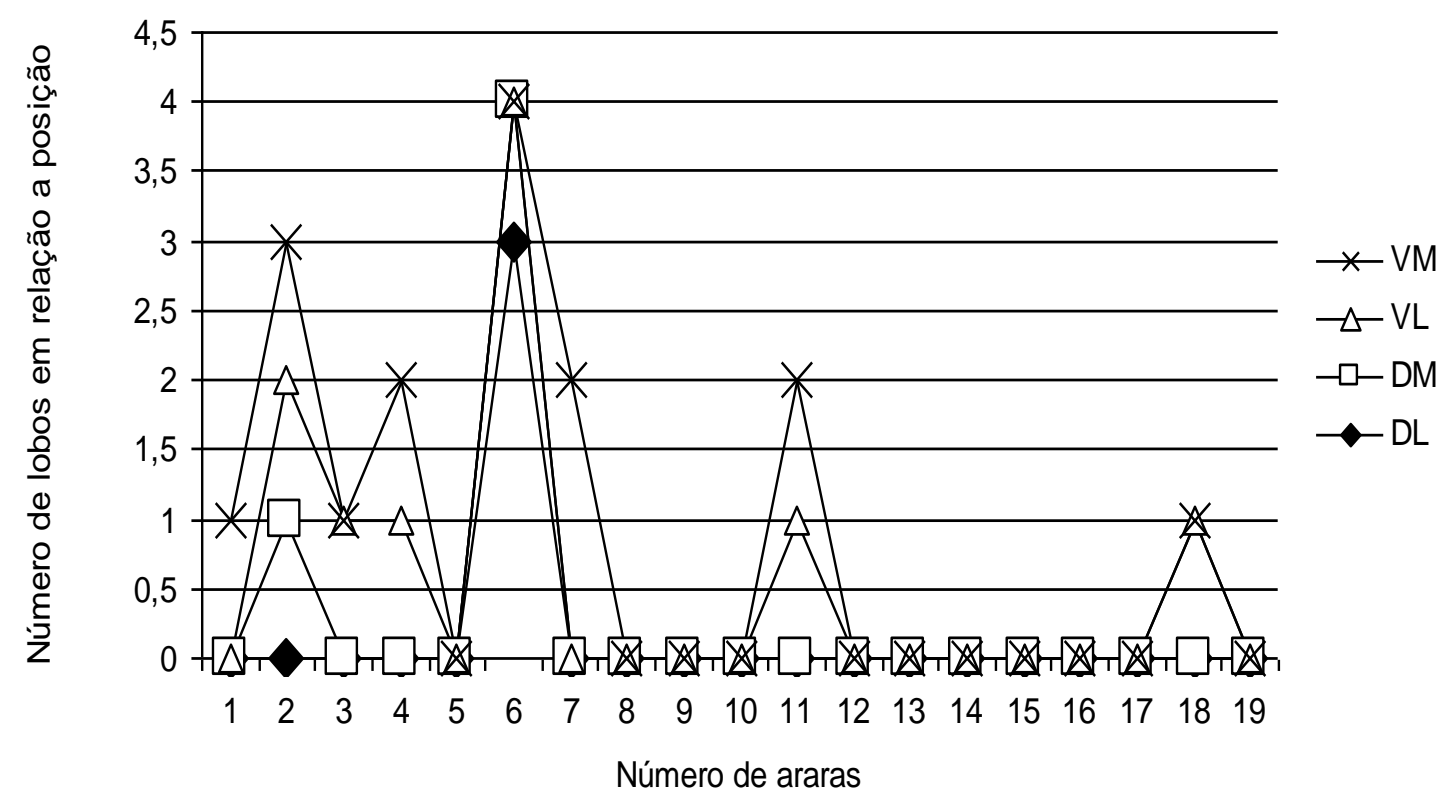

Figura 5. Número dos lobos tímicos cervicais, de acordo com as posições relativas ao plexo vasculoneural. Números de um a 12 correspondem às araras da espécie Ara arauna, de 13 a 17 às araras da espécie Ara chloropterus e de 18 a 19 às araras da espécie Anodorhynchus hyacinthinus. 


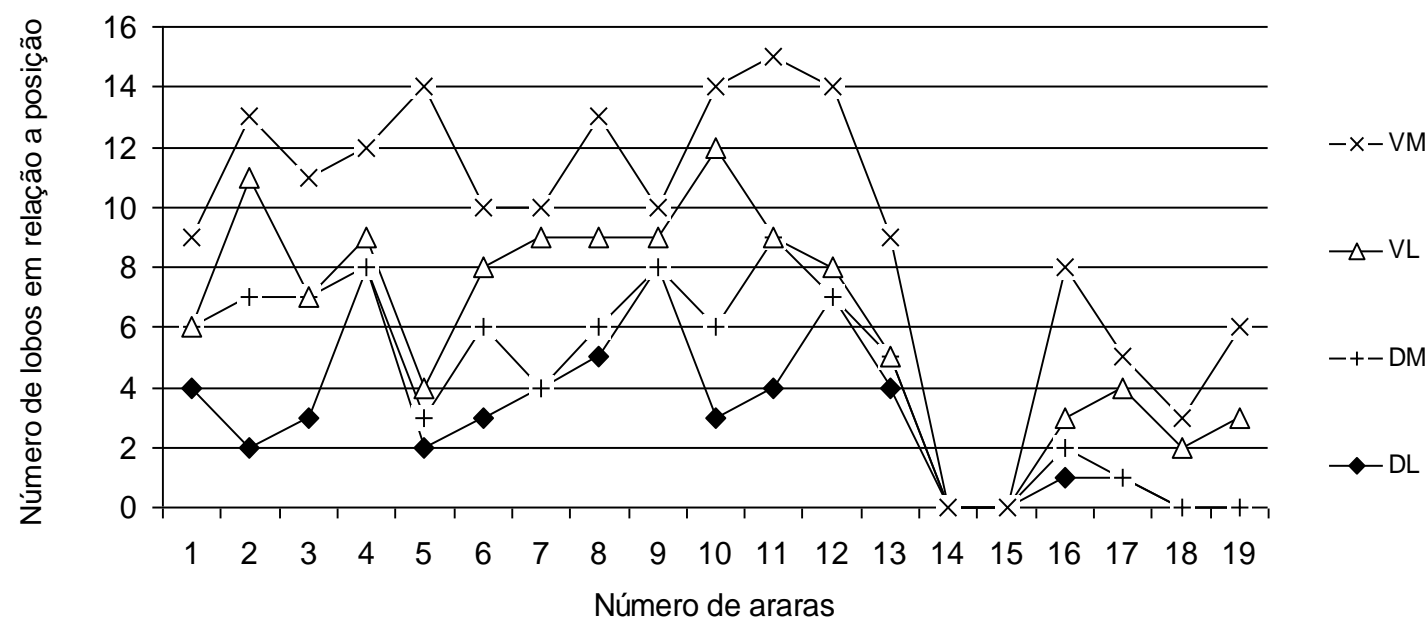

Figura 6. Número dos lobos tímicos celomáticos, de acordo com as posições relativas ao plexo vasculoneural. Números de um a 12 correspondem às araras da espécie Ara arauna, de 13 a 17 às araras da espécie Ara chloropterus e de 18 a 19 às araras da espécie Anodorhynchus hyacinthinus.

Com relação à biometria dos lobos em araras, não foi levado em consideração o fato de serem cervicais ou celomáticos, por serem metricamente muito semelhantes. Assim, independentemente do gênero, os lobos tímicos do antímero direito apresentaram, em média, comprimento de $0,54 \mathrm{~cm}$, largura de $0,16 \mathrm{~cm}$ e espessura de $0,07 \mathrm{~cm}$, enquanto os lobos tímicos do antímero esquerdo foram aferidos medindo $0,45 \times 0,12 \times 0,05 \mathrm{~cm}$.

Especificamente, as araras-canindés apresentaram, em média, os lobos tímicos direitos com $0,60 \times 0,20 \times 0,09 \mathrm{~cm}$ e os lobos tímicos esquerdos com 0,53 x 0,16 x $0,07 \mathrm{~cm}$. Nas araras-vermelhas, os lobos tímicos direitos mediram $0,39 \times 0,08 \times 0,04 \mathrm{~cm}$ e os esquerdos $0,28 \times 0,05 \times 0,02 \mathrm{~cm}$, enquanto nas araras-azuis, os lobos tímicos direitos apresentaram 0,57 x $0,09 \times 0,03 \mathrm{~cm}$ e os esquerdos $0,35 \times 0,02 \times$ $0,0 \mathrm{~cm}$.

Quanto ao peso, ficou estabelecido que, independentemente do gênero e da posição ocupada, os lobos tímicos pesaram, em média, $0,076 \mathrm{~g}$ para os do antímero direito e $0,073 \mathrm{~g}$ para os do antímero esquerdo.

Deve-se ressaltar que, para as diferentes espécies de araras estudadas, os maiores ou menores lobos podem estar distribuídos em qualquer das três porções do pescoço - cranial, médio e caudal.
Também, lobos menores foram observados preferencialmente posicionados ventrolateral ou ventromedial ao plexo vasculoneural, enquanto lobos maiores ocuparam principalmente as posições dorsolateral ou dorsomedial.

\section{DISCUSSÃO}

Estudos concernentes à morfologia do timo das aves têm sido realizados na última década (Nascimento, 2002; Santana et al., 2007) com o intuito de estabelecer um padrão vascular e biométrico, para que possam ser utilizados por pesquisadores de outras áreas como elementos de comparação, pois dados morfológicos, como o peso e o tamanho do timo, são excelentes indicadores do estado de saúde das aves e, portanto, poderão ajudar no melhor entendimento de enfermidades infecciosas e da capacidade de resistências a elas (Milicevic et al., 2002; Marín et al., 2004).

Deve-se levar em conta, também, que o interesse pela pesquisa do timo tem aumentado em virtude da importância que o órgão vem assumindo (Agreste et al., 2007) e da escassez de literatura relativa ao órgão em aves, especialmente às pertencentes à avifauna brasileira (Carvalho, 2003).

Semelhantemente à galinha-d'angola (Onyeanusi et al., 1994; Santana et al., 2007), foi observada 
a ausência de lobos tímicos cervicais e celomáticos em alguns exemplares de araras dissecadas, a qual, de acordo com Hönh (1956) e Anderson (1970), principalmente para os lobos cervicais, pode ser entendida como uma regressão fisiológica, podendo sugerir que, assim como o ovário e os testículos das aves, o timo também pode, conforme a época do ano, aumentar ou diminuir de tamanho e peso, especialmente durante o período reprodutivo ou durante a fase de muda de penas (Ward e Kendall, 1975; Dawson et al., 2001).

Ainda, a comparação de tamanho entre os lobos tímicos cervicais das araras estudadas com os encontrados em galiformes permite identificar que os lobos são, em média, quase $50 \%$ menores, fato que também contribuiu para o desenvolvimento dessa hipótese de regressão fisiológica.

Quanto à descrição topográfica dos lobos cervicais frente ao plexo vasculoneural, ficou evidente que a presença de um fascículo conjuntivo envolvendo os lobos cervicais, bem como a variação de tamanho deles (Baumel, 1993; Carvalho, 2003; Lima et al., 2004; Santana et al., 2007; Santana et al., 2008; Arantes, 2010), pode modificar a posição ocupada por eles, pois, como demonstrado nas dissecações realizadas, lobos menores tendem a ocupar posições mais ventrolaterais em relação ao plexo vasculoneural, fato condizente com os achados de Onyeanusi et al. (1994), enquanto lobos maiores tendem a ocupar uma posição mais dorsolateral (Nascimento, 2002; Santana et al., 2008).

Além do fascículo conjuntivo, também foi observada uma fina camada de gordura situada superficialmente aos lobos, e a maneira como esta gordura estava disposta entre um lobo e outro permitiu caracterizá-los como estruturas anatômicas individuais, pois cria-se espaço suficiente para isolá-los, sendo esta uma característica diferencial pertinente entre as aves e os mamíferos (Arantes, 2010).

Ainda, a posição dos lobos tímicos cervicais observados preferencialmente no terço cranial do pescoço das araras dissecadas também pode estar associada com a hipótese de regressão fisiológica proposta, já que este posicionamento não foi constatado para nenhum galiforme, inclusive em idade adulta (Nascimento, 2002).
Quanto ao número médio de 4,6 lobos tímicos cervicais encontrados por antímero nas diferentes espécies de araras, nota-se uma variação muito grande com relação à média de 13 lobos encontrados para galiformes, anatídeos, cracídeos e pombos (Nascimento, 2002; Carvalho, 2003; Lima et al., 2004; Santana et al., 2004).

Para tentar entender essa diferença, deve-se levar em consideração a ideia de regressão fisiológica proposta, pois, como ponderado por Santana et al. (2007), que realizaram uma revisão da literatura pertinente ao assunto, existe grande probabilidade de que o número médio de lobos tímicos seja muito semelhante entre os diferentes gêneros de aves, apesar de variações individuais nos valores máximo e mínimo serem comumente observadas.

A grande variação observada para o número de lobos celomáticos encontrados nas araras dissecadas e nos relatos de Carvalho (2003) e Lima et al. (2004) para pombos e patos, quando estes foram confrontados com o gênero Gallus, não é muito divergente, já que normalmente estas aves não possuem tais lobos (Nascimento, 2002; Santana et al., 2007; Santana et al., 2008).

A presença de lobos celomáticos nas araras dissecadas e nos descritos para pombos, patos e mutuns (Carvalho, 2003; Lima et al., 2004; Santana et al., 2004), principalmente no antímero direito, pode estar relacionada diretamente com a presença do inglúvio, que se posiciona nestas espécies de maneira muito próxima à abertura cranial da cavidade celomática, o que pode dificultar o posicionamento de lobos cervicais dorsalmente a ele, pelo seu grande volume, bem como pela estreita abertura cranial da cavidade celomática observada nestas espécies (Santana $e t$ al., 2007).

Outro parâmetro observado nas araras dissecadas foi o peso dos lobos tímicos, porém não foi possível sua comparação com outros gêneros de aves, pois o mesmo parâmetro não foi elemento de estudo para Carvalho (2003), Lima et al. (2004) e Santana et al. (2004), os únicos que trabalharam com aves silvestres.

Quanto ao descrito por Nascimento (2002) e Santana et al. (2007) referente à penetração do tecido tímico pela glândula tireoide e quanto ao 
exposto por Santana et al. (2001), a grande variabilidade de tamanho e a proximidade topográfica apresentada pelos órgãos nas araras dissecadas podem aumentar as chances desta interação, principalmente quando tratados os lobos tímicos posicionados mais caudalmente ao pescoço.

\section{CONCLUSÕES}

Nas diferentes espécies de araras, os arranjos topográfico e de distribuição dos lobos tímicos apresentaram-se de forma muito similar ao encontrado para outras espécies de aves domésticas, apesar de serem menores. Mesmo com o pequeno número de aves estudadas, a ausência de lobos tímicos cervicais verificada em $10 \%$ dos casos estudados, juntamente com a grande variação de tamanho e número observados nas araras dissecadas, sugere uma regressão fisiológica do órgão, principalmente em aves sexualmente maduras, apesar de essa premissa necessitar de maiores estudos.

\section{AGRADECIMENTOS}

Ao Criadouro Poços de Caldas, na figura do Sr. Moacir Carvalho Dias, por fornecer e permitir a utilização do material para a realização deste trabalho, favorecendo o aprimoramento da anatomia comparativa.

\section{REFERÊNCIAS}

AGRESTE, F.R.; BOMBONATO, P.P.; HERNANDEZ-BLASQUEZ, F.J. Características morfométricas do desenvolvimento do timo em cães. Pesq. Vet. Bras., v.27, p.236-240, 2007.

ANDERSON, W.L. Seasonal changes in thymus weights in Red-neckd pheasants. Condor, v.72, p.205-208, 1970.

ARANTES, R.C. Vascularização arterial do timo em galinhas caipiras (Gallus gallus). 2010. 49f. Dissertação (Mestrado Anatomia dos Animais Domésticos) - Faculdade de Medicina Veterinária, Universidade Federal de Uberlândia, Uberlândia, MG.

BAUMEL, J.J. Handbook of avian anatomy: nomina anatomica avium. 2.ed. Cambridge: Nuttall Ornithological Club, 1993. 779p.
BOMBONATO P.P. Aspectos da morfologia, topografia e vascularização arterial do timo em fetos de búfalo. 1997. 88f. Tese (Livre Docência) - Faculdade de Medicina Veterinária e Zootecnia, Universidade de São Paulo, São Paulo.

CARVALHO, V.B. Morfologia e morfometria do timo em pombos domésticos (Columba livia gmelin). 2003. 89f. Trabalho de Conclusão de Curso (Graduação em Ciências Biológicas) Curso de Ciências Biológicas, Universidade de Franca, Franca.

DAWSON, A.; KING, V.M.; BENTLEY, G.E. et al. Photoperiodic Control of Seasonality in Birds. J. Biol. Rhythms, v.16, p.365-380, 2001.

ERCOLINI, C.B. Estudo quantitativo da vascularização do timo em gatos. 2007. 81f. Dissertação (Mestrado em Anatomia dos Animais Domésticos) - Faculdade de Medicina Veterinária e Zootecnia, Universidade de São Paulo, São Paulo.

HÖHN, E.O. Seasonal recrudescence of the thymus in adult birds. Can. J. Biochem. Physiol., v.34, p.90-101, 1956.

LIMA, R.Z.; SANTANA, M.I.; DOS REIS, I.A. et al. Morfologia e vascularização do timo em patos (Anas platyrhynchus). In: REUNIÃO DE INTEGRAÇÃO DA MORFOLOGIA PANAMERICANA, 2004, Foz do Iguaçu. Anais... Foz do Iguaçu: 2004. p.12. (Resumo).

MARÍN, F.P.; NAVA, J.; MAVÁREZ, Y. et al. Caracterización morfométrica de los órganos linfoides en pollos de engorde de la línea Ross criados bajo condiciones de campo en el estado Zulia, Venezuela. Rev. Cient., v.14, p.217-225, 2004.

MILICEVIC, Z.; ZIVANOV, V.; MILICEVIC, N.M. Involution of bursa cloacalis and thymus in ciclosporin A - treated chickens. Anat. Hist. Embriol., v.31, p.61-64, 2002.

NASCIMENTO, M.A. Vascularização arterial do timo em aves (Gallus gallus domesticus, Linnaeus, 1758) da linhagem Cobb. 2002. 91f. Dissertação (Mestrado em Anatomia dos Animais Domésticos) - Faculdade de Medicina Veterinária e Zootecnia, Universidade de São Paulo, São Paulo. 
ONYEANUSI, B.I.; ONYEANUSI, J.C.; EMMA, A.N. et al. The thymus of the guinea fowl from the eighteenth day of incubation until maturity. Anat. Histol. Embryol., v.23, p.320-329, 1994.

SANTANA, M.I.S.; SILVA, F.O.C.; SEVERINO, R.S. et al. Vascularização arterial do timo em aves (Gallus gallus domesticus - Linnaeus, 1758) da linhagem NPK. Vet. Not., v.7, p.13-19, 2001.

SANTANA, M.I.; NETTO, A.P.; SILVA, F.O.C. et al. Morfologia e irrigação do timo em mutuns (Crax fasciolata). In: REUNIÃO DE INTEGRAÇÃO DA MORFOLOGIA PANAMERICANA, 2004, Foz do Iguaçu. Anais... Foz do Iguaçu: 2004. p.14. (Resumo).
SANTANA, M.I.; BOMBONATO, P.P.; SILVA, F.O.C. et al. Morfologia e morfometria do timo em galinhas da angola (Numidea meleagris galeata). RPCV, v.102, p.43-48, 2007.

SANTANA, M.I.; BOMBONATO, P.P.; ARAÚJO, L.V. et al. Irrigação do timo em aves da linhagem Paraíso Pedrês (Gallus gallus domesticus). Arq. Bras. Med. Vet. Zootec., v.60, p.307-314, 2008.

WARD, P.; KENDALL, M.D. Morphological changes in the thymus of young and adult Redbilled queleas Quelea quelea (Aves). Phil. Trans. Rev. Soc. B, v.273, p.55-64, 1975. 